An Atlas of Stellar Spectra

With an Outline of Spectral Classification. By W. W. Morgan, Philip C. Keenan and Edith Kellman. (Astrophysical Monographs, sponsored by the Astrophysical Journal.) Pp. vi $+36+55$ plates. (Chicago : University of Chicago Press; London : Cambridge University Press, 1943.) Price 60s. net.

T. HIS much-needed atlas defines a two-dimensional classification system of stellar spectra by means of a sequence of standard spectra, many of which are illustrated in the fifty-five photographic prints form. ing the main body of the work. The spectra were taken specially for the purpose on a small one-prism spectrograph attached to the 40-in. Yerkes refractor. The standard stars chosen, which are mostly brighter than the eighth apparent magnitude, are of types from $O 5$ to $M 2$, and were selected to exhibit a large range of luminosities. A given spectrum is considered uniquely classified when its spectral type (one of the conventional seven classes subdivided decimally) and its luminosity (one of five classes ranging from supergiants to the main sequence) are specified.

The "Outline" accompanying the prints gives a brief review of the taxonomic principles involved, and goes on to describe the criteria employed for both co-ordinates of the classification, and to tabulate the standard stars. No originality is claimed either for the system defined or for the criteria used: of necessity they are both evolved from work done, mainly at Harvard and Mount Wilson, by many previous investigators. The spectral types are closely correlated with colour, and will thus be invaluable in studies of interstellar absorption. The luminosities, when calibrated, will make possible the determination of accurate spectroscopic parallaxes on low-dispersion spectrograms.

The prints themselves are excellently reproduced and show well the lines, often quite faint, which are used for the classification of spectral type or lumin. osity. They are negative prints and can thus be compared directly with spectrograms in the observatory. The descriptions, though concise and lucid, are reproduced in a manual script which does not enhance the appearance of the Atlas, but the "Outline" is printed in the impeccable tradition of the "Astrophysical Monographs". It is not too much to predict that this work will quickly become a tool indispensable to every practical astrophysicist.

\section{The Earth and Man}

A Human Geography. By Prof. Darrell Haug Davis. Pp. xxiii +675 . (New York : The Macmillan Company, 1942.) 21s. net.

A.S geographers become more agreed on their A province of study, they approach nearer to what might well be termed human ecology and find a field of observation that has been largely neglected in other studies. The analytical outlook, in geography as in other sciences that deal with living organisms, gives place to the more difficult synthetic outlook, which in geography is one of great complexity, because man is more concerned in improving, or at least in changing, his environment than in being dominated by it. In human ecology, the organism's reaction is far greater than in plant and animal ecology, and the risk of neglecting human influence on surroundings is a constant danger.

Prof. Davis has written a careful, though not profound, introduction to this aspect of geography, but he claims no further aim than the writing of a text-book for the early stages of university work, and this no doubt explains his general avoidance of problems in social geography. He has produced an admirable survey of the factors that decide the grouping and pattern of human distributions with special reference to North America. The rest of the world-especially the monsoon lands of Asia-are not neglected, but the book is primarily for American students. It is admirably illustrated with maps, diagrams and photographs, and has a full index. The bibliographical references might well be on a wider scale.

R. N. R. B.

\section{The Bushman Tribes of Southern Africa}

By A. M. Duggan-Cronin; with an Introductory Article on the Bushman Tribes and Descriptive Notes on the Plates, by D. F. Bleek. Pp. v $+15+40$ plates. (Kimberley: Alexander McGregor Memorial Museum, 1942.)

ATHOUGH the actual text of this book com$A$ prises only 14 pages, a great deal of reliable and interesting information is given on the physical characters and culture of the Bushman tribes. This information is supplemented by a useful map showing the distribution of the various tribal units. It would, perhaps, have been a useful addition to have provided a short key to the rather baffling system of phonetics employed in writing native words. Condensation was naturally necessary in so brief an introduction, but this brevity is in certain cases rather unsatisfactory. It is tantalizing to be told that : "In all tribes they make rain in some manner", but to be given no hint as to the proeedure adopted in any tribe.

The story of these people is a sad one : "They are being gradually forced into servitude of black or white men, and absorbed into the coloured population of South Africa". The forty plates, most admirably reproduced from excellent photographs, constitute a permanent and valuable record of these doomed people. In them are preserved scenes from their daily life, records of many of their social and cultural activities and the physical characters of certain well-selected types. The National Research Board of South Africa and the authorities of the Alexander McGregor Memorial Museum are to be congratulated on the production of a publication of such perfection in the year 1942.

F. WOOD JONES.

The Cathode Ray Oscillograph in Incustry By Dr. W. Wilson. Pp. xii $+150+42$ plates. (London: Chapman and Hall, Ltd., 1943.) I2s. 6d. net.

$\triangle$ PPLICATIONS of the sealed-off cathode-ray tube to the field of radio have been previously well treated, so the author gains by concentrating on other applications, which are shown to be wide and valuable. Controlling electron beams in' cathode-ray tubes, electron diffraction tubes, and electron microscopes exhibits many common features, and the author brings these and related topics up to date in book-form. He also shows that the sealed-off glass tubes and the metal continuously evacuated tubes have their separate fields of application and are not always competitors. He gives some of the latest electron microscope photographs, and shows how essential this new instrument is in many fields of research. It is unfortunate that the War has further retarded the development of electron microscopes in Great Britain, an opportunity which even private generosity has neglected. The text is well produced considering the present conditions, and includes the essential references. L. E. C. HuGhes. 